\title{
Experimental Determination of the Parameters for Modelling Semi-Solid Processing
}

\author{
$\mathrm{H} \vee$ Atkinson \\ Head of Mechanics of Materials Group, Department of Engineering, University of Leicester, \\ University Rd., Leicester, LE1 7RH, UK \\ Hva2@le.ac.uk
}

Keywords: Rheometry; compression; modelling semi-solid processing; rapid transients; steady state.

\begin{abstract}
The numerical modeling of semi-solid processing requires data on the rheological properties of materials. This data is often obtained by rheometry but there are difficulties with characterizing high solid fractions, where the torque which can be exerted with the rheometer is insufficient. A number of other methods for measuring the flow parameters, including compression between platens, have been utilized. The various methods will be reviewed in this paper. Computational fluid dynamics modelers have often used data from steady state experiments but it is the behaviour during rapid transients which is more relevant to the actual semi-solid processing route.
\end{abstract}

\section{Introduction}

At MIT in the early 1970s, Spencer at al. [1] discovered that metallic alloys in the semi-solid state with a non-dendritic microstructure, (i.e. spheroids of solid in a liquid matrix), behaved in a thixotropic way. When sheared the material thinned but when it was allowed to stand it thickened again. This occurs because, on shearing, the bonds between spheroids, and between agglomerates of those spheroids, are broken down and when allowed to stand they rebuild. This behaviour is the basis of a family of innovative manufacturing processes termed semi-solid processing. The technology is in use commercially in the form of:- thixoforming, where material with a nondendritic microstructure on reheating into the semi-solid state is forced into a die; rheocasting, where a liquid alloy is cooled into the semi-solid state in such a way as to give a non-dendritic microstructure, and then placed in the shot sleeve of a die casting machine; and thixomoulding (which to date has only been applied to magnesium alloys), which is allied to injection moulding of polymers, with a continuous screw feed. In all cases, die design can be made more efficient and effective through the use of modelling. Modelling requires the input of parameters to describe the rheological behaviour of materials. The purpose of this paper is to review the experimental determination of these parameters. A fuller discussion, extensive references and full details of the equations are given elsewhere [2]. Previous reviews include those by Kirkwood [3], Atkinson [4], Alexandrou [5], Fan [6] and Modigell [7].

It is necessary first to give some background rheology and then to look at the measurement of flow under a steady shear rate and flow under a changing, (i.e.transient) shear rate.

\section{Background Rheology}

In a Newtonian fluid, the shear stress, $\tau$, is proportional to the shear rate, $\dot{\gamma}$, and the constant of proportionality is the viscosity, $\eta$. Thixotropic fluids are non-Newtonian i.e. the shear stress is not proportional to the shear rate. The viscosity is then termed the apparent viscosity and is dependent on shear rate, pressure, temperature and time. Some non-linear fluids also show viscoelasticity i.e. they store some of the mechanical energy as elastic energy. Thixotropic materials do not store energy elastically and show no elastic recovery when the stress is removed. 
If a fluid exhibits a yield stress and then gives a linear relationship between shear stress and shear rate, it is termed a Bingham material. Then:

$$
\tau=\tau_{y}+k \dot{\gamma}
$$

where $k$ is a constant related to the viscosity. The Herschel-Bulkley model is where behaviour is non-linear after yield i.e.:

$$
\tau=\tau_{y}+k \dot{\gamma}^{n}
$$

There is dispute over whether thixotropic semi-solid alloys display yield [eg. 8] and whether they should be modelled as such (e.g. [5]). Koke and Modigell [9] have used a shear stress controlled rheometer to measure yield stress directly on $\mathrm{Sn} 15 \% \mathrm{~Pb}$. They distinguish between a static yield stress where the fluid is at rest prior to the application of a shear stress, and a dynamic yield stress where the fluid is being continuously sheared. Their results are shown in Fig. 1.
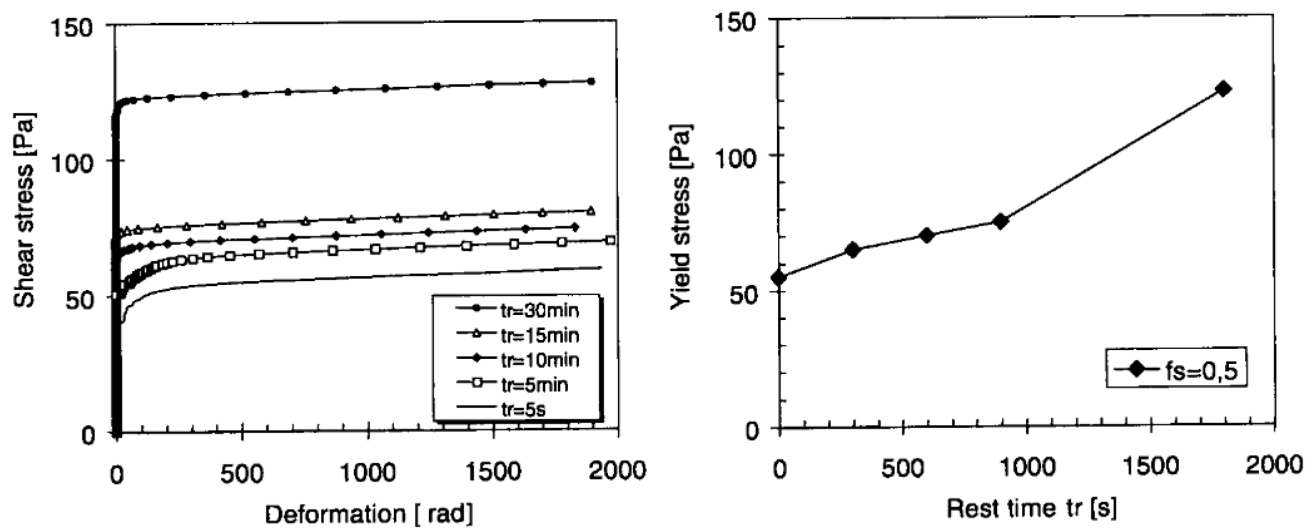

Fig. 1 Shear-stress ramp experiments after different rest times (tr) for $\mathrm{Sn}-15 \% \mathrm{~Pb}$ [9]. (a) Shear stress versus deformation angle. (b) Shear stress versus rest time. Temperature $195^{\circ} \mathrm{C}$, fraction solid 0.5 , globular structure prepared by shearing at $100 \mathrm{~s}^{-1}$ at a cooling rate of $1{ }^{\circ} \mathrm{C} / \mathrm{min}$.

The yield stress increases with rest time prior to deformation because of the increasing degree of agglomeration. In terms of modelling semi-solid alloy die fill, the use of a yield stress may be appropriate because a vertical billet does not collapse under its own weight unless the liquid fraction is too high. In addition, in rapid compression experiments (to be described) later an initial peak in the load versus displacement curve is detected. Contrary to this, though, is the fact that, at the 'thixoforming temperature', the initial peak is so small as to be undetectable.

The Ostwald de Waele relationship:

$$
\tau=k \dot{\gamma}^{n}
$$

is used to describe fluids which do not have a yield point and where there is a power law relationship between the shear stress $\tau$ and the shear rate $\dot{\gamma}$. If the exponent $n=1$, this reduces to the expression for a Newtonian fluid with the constant $k$ equal to the viscosity $\eta$. A shear thinning material (whose viscosity decreases as the shear rate increases) would have a value of $n$ of less than 1 and a shear thickening material would have $n$ greater than one. Thixotropic materials are essentially shear thinning but also thicken again when allowed to rest (i.e. all thixotropic materials are shear thinning but not all shear thinning fluids are thixotropic). 
It is thought that at very high shear rates and at very low shear rates, thixotropic fluids effectively become Newtonian. This is expressed in the Cross model:

$$
\eta=\eta_{\infty}+\left[\frac{\eta_{0}-\eta_{\infty}}{1+k \dot{\gamma}^{n}}\right]
$$

where as the shear rate $\dot{\gamma} \rightarrow 0, \eta \rightarrow \eta_{0}$ and as $\dot{\gamma} \rightarrow \infty, \eta \rightarrow \eta_{\infty}$. These are steady-state viscosities and, as will be discussed below, it is the transient behaviour which is particularly of importance for the modelling of thixotropic die fill.
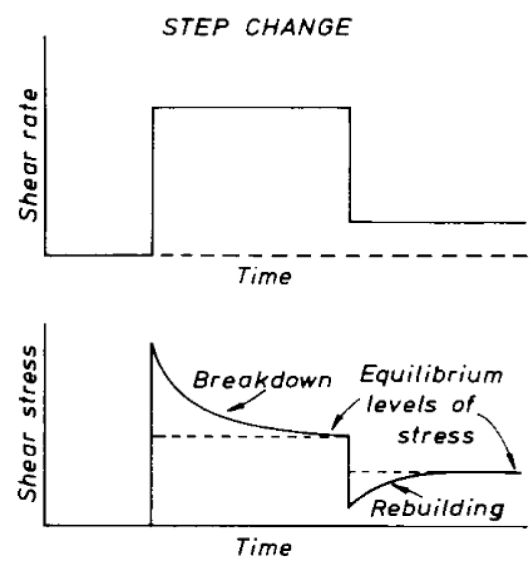

Fig 2. Response of an inelastic thixotropic material to first a step-change up in shear rate and then a stepchange down..
Viscosity is highly dependent on temperature. For a Newtonian fluid (e.g. the liquid matrix in a semisolid slurry), the viscosity decreases with increase in temperature. Temperature also affects the microstructure. Thus, in semisolid slurries, the fraction solid decreases with increase in temperature, with a consequent effect on viscosity. In addition, over time, the microstructure will coarsen by diffusion and this will be accelerated as the temperature increases. Quite a lot of data is available for the model $\mathrm{Sn} / \mathrm{Pb}$ alloys but there is less for aluminium alloys because there are few commercially available rheometers that operate above about $500^{\circ} \mathrm{C}$.

For a thixotropic material at rest, when a step increase in shear rate is imposed, the shear stress will peak and then gradually decrease until it reaches an equilibrium value for the shear rate over time (Fig. 2). The higher the shear rate after the step, the lower the equilibrium viscosity. The peak viscosity encountered will increase with increasing rest time before it recovers back to the equilibrium viscosity of the shear rate specified.

\section{Steady State Measurements}

The flow behaviour in the steady state is dependent on the fraction solid, particle size, particle shape, time and stress history. It can also depend on the temperature history; the results will be different if the semi-solid state originated from cooling from the liquid state or reheating from the solid state. This is because of Ostwald ripening of the solid spheroids during the treatment, which is turn leads to a change in particle size.

There are three principal methods of carrying out steady state measurements. These are capillary rheometry; rotational rheometry and compression experiments. Capillary rheometers, where material is pushed down a tube and the pressure drop measured, give high shear rates and conditions close to those during semi-solid processing. However, measuring the pressure drop accurately can be difficult.

Rotational rheometry with concentric cylinder rheometers has been widely used but can generally only work with fractions of solid below about 0.5 ; above this the material is too stiff for the instrument to operate. In addition, in the past, few such rheometers have been capable of operating at temperatures where Al alloys are semi-solid, or above. This situation is now changing (e.g. $[10,11])$. The shear rates available in rotational rheometers are in the medium range.

To examine slurries with fractions of solid above 0.5 there are various options. Laxmanan and Flemings [12] established a technique involving slow compression. The material is placed between two insulating platens (for isothermal conditions) and the load measured during deformation at constant velocity. The results are difficult to interpret because the deformation is inhomogeneous (in 
contrast with the steady state in a viscometer). Laxmanan and Flemings assume, for simplicity, in their analytical solution, that the flow is in fact Newtonian. This is clearly a shortcoming. A step further would be to use Computational Fluid Dynamics (CFD) to simulate the compression but this would need to be an iterative process because the CFD, in itself, requires the assumption of a rheology model and the input of parameters.

Other potential methods include forward extrusion [e.g. 13], although the tests may not be isothermal and there is a possibility that only material in the wall region may be broken down leading to 'plug' flow. However, appropriate design of the die entrance can break the material down and there is reasonable agreement between the results of these workers and those from earlier experiments by slow compression [12].

\section{Transient Behaviour}

Computational Fluid Dynamics (CFD) has been extensively used to predict die filling (see eg. [2]). However, some of the work reported has been based on rheological data obtained in steady state experiments, where the semi-solid material has been maintained at a particular shear rate for some time. In reality, in thixoforming for example, the slurry undergoes a sudden increase in shear rate from rest to $100 \mathrm{~s}^{-1}$ or more as it enters the die. This change takes place in less than a second. Hence, measuring the transient rheological response under rapid changes in shear rate is critical to the development of modelling of die filling and successful die design for industrial processing. It can be investigated with two types of experiment. Firstly, via rapid shear rate changes in a rheometer and secondly, for higher fractions solid (where the torque capability of a rheometer is not sufficient), with rapid compression experiments, for example, in the thixoformer itself or in a drop forge viscometer.

Rapid Shear Rate Changes in Rheometers Studies of transient behaviour have included those by Kumar [14], Quaak [15], Peng and Wang [16], Mada and Ajersch [17], Azzi et al [18] Koke and Modigell [9] Modigell and Koke [19,20] and Liu et al. [21,22]. Two relaxation times were quantified: (1) breakdown time and (2) buildup time. The breakdown time is the characteristic time for the slurry to achieve its steady-state condition after a shear rate change from a lower value to a higher value, while the buildup time is for a change from a higher shear rate to a lower shear rate. These workers found that the times for breakdown are faster than those for buildup. This would be expected, as the breaking up of 'bonds' between spheroidal solid particles in agglomerates is likely

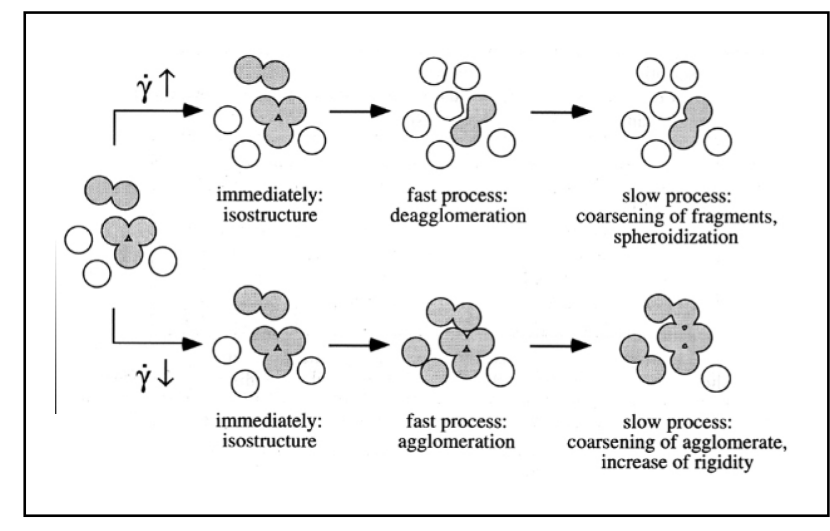

Fig. 3 Schematic model describing the fast and slow processes in a semi-solid material's structure after shear rate up and down jumps (taken from ref. [15]) to be easier than the formation of bonds during shear-rate drops. Quaak [15] proposes two characteristic times to describe a shear rate jump. He suggests that during a shear-rate change, the slurry undergoes an initial rapid breakdown/buildup followed by a more gradual process dependent on diffusion. This can be described by a double exponential expression. Quaak gives Fig.3 as the microstructural basis. Immediately after a change in shear rate, the structure remains the same ('iso-structure'). This is followed by a very fast process and then a slow process, associated with diffusion, giving coarsening and spheroidisation. It is the "very fast process' which is relevant to modelling die fill. In a rheometer, great care must be taken to ensure that inertial effects do not interfere with the 
results [e.g. see 22]. In addition, instrumental effects must be carefully separated from those of the material itself, particularly when attempting to examine behaviour that occurs in less than a second. For example, electronic switching may occur during the shear rate jump. This can be allowed for by only analysing results after the shear rate has reached $\sim 90 \%$ of the specified final shear rate (see [22]). The work of Liu et al. [22] involves the fastest data collection rate so far $(\sim 1 \mathrm{kHz}$ capture rate). This is significantly faster than that used by other workers $(200 \mathrm{~Hz}$ in [14], $9 \mathrm{~Hz}$ in [15], 200 $\mathrm{Hz}$ in [16]) and enables the capture of the very fast process. The results for shear rate jumps from 0 to $100 \mathrm{~s}^{-1}$ after different rest times are shown in Fig. 4. With longer rest times, the peak stress recorded increases. The breakdown times in Table 1 were obtained by fitting an exponential to the data obtained during the second after $90 \%$ of the final shear rate was achieved. In Table $1, \eta_{\mathrm{p}}$ is the peak-stress viscosity, $\eta_{\mathrm{ss}}$ is the 'first' steady-state viscosity (given that there are at least two processes going on as mentioned earlier) and $\tau_{b}$ is the 'first' breakdown time. Table 1 shows that the longer the rest time prior to the shear rate jump, the lower the breakdown time. This is consistent with microstructural evidence showing that increasing the rest time increases the solid-particle sizes and the degree of agglomeration. This increase would impede the movement of the particles upon the imposition of the shear stress. The ease with which particles are able to move past each other depends on the fraction of liquid medium present, the size of the particles and the degree of agglomeration. The data show, that during a change in shear rate, in about 0.15 seconds the semisolid structure would have broken down from its initial state. Regardless of the initial shear rate, the breakdown time decreases with increasing final shear rate $[15,17,18,20,22]$.

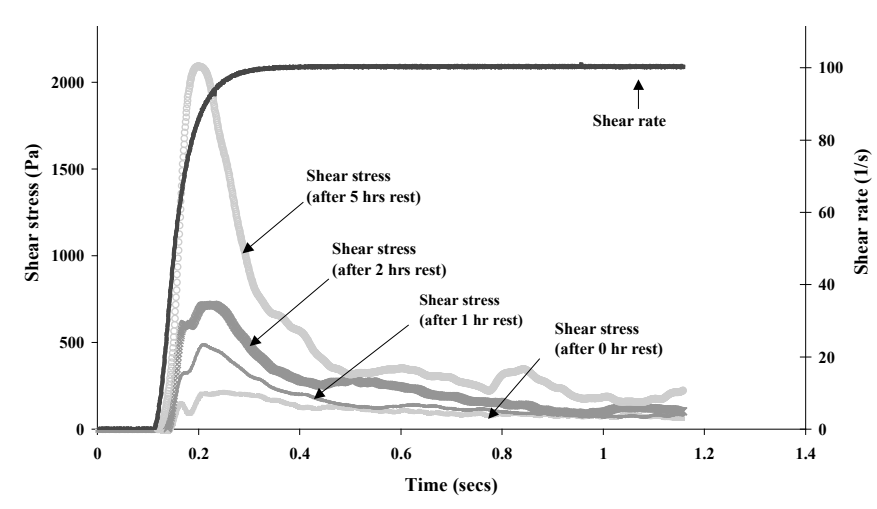

Fig. 4 Shear rate jumps from 0 to $100 \mathrm{~s}^{-1}$ after different rest times for $\mathrm{Sn} 15 \% \mathrm{~Pb}$ alloy at fraction solid 0.36 [22].
Parameters obtained from shear rate jump experiments

\begin{tabular}{|c|c|c|c|c|}
\hline Shear rate & \multicolumn{4}{|c|}{$0-100$} \\
\hline $\begin{array}{c}\text { Rest times } \\
(\mathrm{hrs})\end{array}$ & 0 & 1 & 2 & 5 \\
\hline$\eta_{p}(\mathrm{Pas})^{*}$ & 2.1 & 5.4 & 8.0 & 23.0 \\
\hline $\begin{array}{c}\eta_{s s} \\
(\mathrm{Pas})^{* *}\end{array}$ & 0.8 & 0.8 & 1.2 & 2.0 \\
\hline$\tau_{b}(\mathrm{~s})^{* * *}$ & 0.18 & 0.16 & 0.15 & 0.12 \\
\hline $\begin{array}{l}* \text { The errors ar } \\
0.5) \\
* * \text { The errors a } \\
0.2) \\
* * * \text { The errors } \\
0.03)\end{array}$ & withi & $\%$ con & ace li & $\begin{array}{l}( \pm \\
s( \pm \\
t s( \pm\end{array}$ \\
\hline
\end{tabular}

Table 1: Tabulation of parameters obtained from shear rate jump experiments on $\mathrm{Sn} 15 \% \mathrm{~Pb}$ alloys (at $\mathrm{Fs}=0.36)$ under different rest times $[22]$.

As far as the existence of 'iso-structure' during the jump is concerned, Turng and Wang [23] and Peng and Wang [16] observed an overshoot in the measured stress during a rapid increase in shear rate. They found that this overshoot (or undershoot in the case of a decrease in shear rate) is proportional to the change in shear rate. Therefore, they argue, for that instant, the material is behaving in a Newtonian way. The viscosity, and hence the structure, is constant, during the change. Peng and Wang [16] observed that the overshoot increases with increasing solid fraction. Horsten et al. [24] and Quaak and co-workers [15,25] argued that during this transient period structure evolution has not had time to occur and the structure corresponds to that of the previous shear rate. 
Kumar et al [25] and Koke and Modigell [9] however, find shear thickening 'iso-structural' flow behaviour and the latter authors argue that this finding is of high importance for simulation of the industrial process.

Data on the transient behaviour of aluminium alloys is sparse because the majority of the commercially available rheometers do not operate at semisolid aluminium alloy temperatures.

Rapid Compression For high solid fractions, above about 0.5, conventional rheometers do not have sufficient torque capability. Other methods must then be used, introducing complexity because the shear rate is no longer constant throughout the material (as it can be assumed to be in a concentric cylinder rheometer). Laxmanan and Flemings [12] measured the force and displacement for $\mathrm{Sn} 15 \% \mathrm{~Pb}$ compressed between parallel plates at low strain rates. The resulting load was not measured directly (but rather, derived from the pressure on the ram) and the rate of compression was much slower than in the industrial process. The work of Loué et al [27], carried out at higher shear rates by backward extrusion on aluminium alloys, resembles industrial thixoforming more closely. However, the specimens were heated to temperature over a long period of time $(\sim 10$ minutes) and then held isothermally for 30 minutes before compression. Such time periods would be considered long in industrial thixoforming. Yurko and Flemings [28] designed a drop forge viscometer to study fluid flow behaviour at transient high shear rates. It consists of a lower platen and an upper platen, with an attached platen rod to track platen motion with time. It is similar to a parallel plate compression viscometer but the upper plate is allowed to fall under the influence of gravity. A high-speed digital camera images the rod as it falls. The force is calculated from the second derivative of the displacement data allowing calculation of viscosities at shear rates in excess of $1000 \mathrm{~s}^{-1}$. A typical experiment yields instantaneous, volume-averaged viscosity first under rapidly increasing shear rate and then under rapidly decreasing shear rate. Segregation of liquid from solid did not occur at the high shear rates.

Liu et al. [29] have carried out rapid compression in the thixoformer itself using a load cell to record the load versus time signals. The compression rate is then akin to industrial thixoforming and the load is measured directly. A series of typical signal responses is shown in Fig. 5.

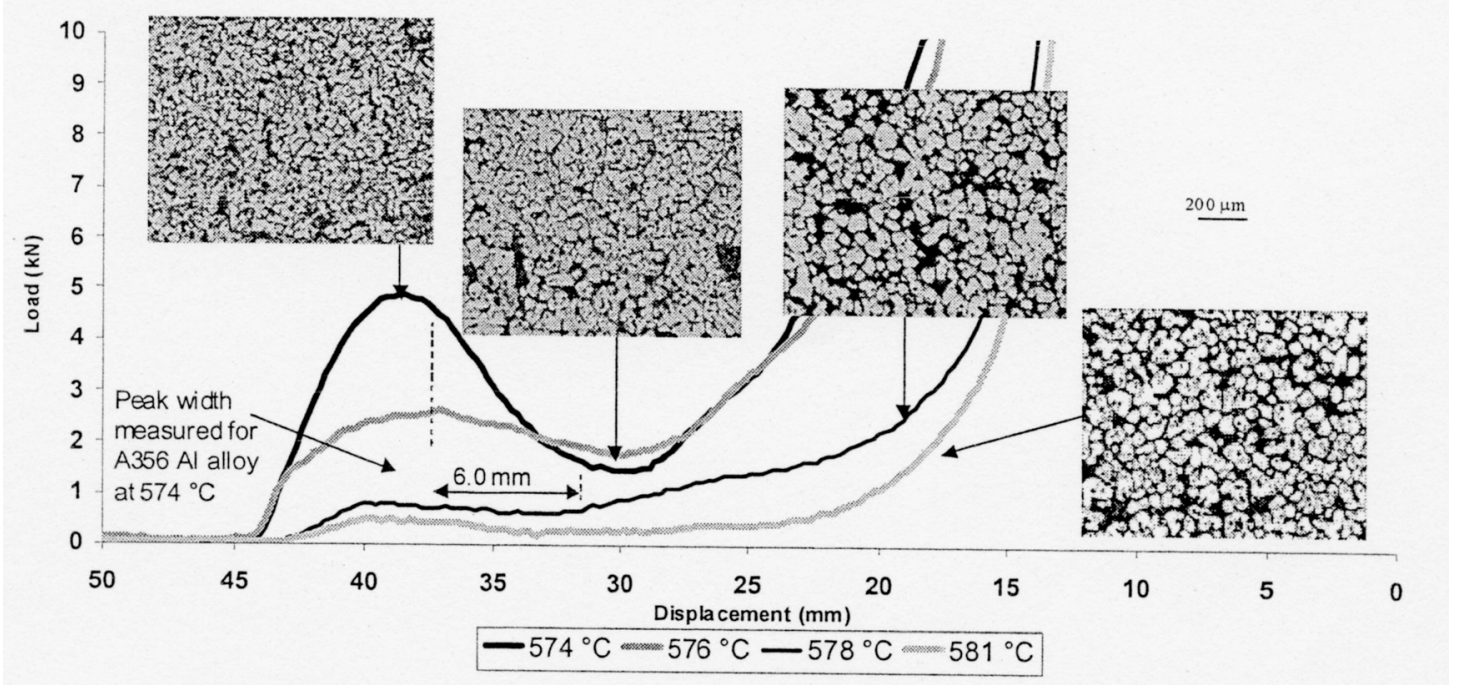

Fig. 5 Load signals and microstructures at different temperatures for Alusuisse A356 aluminium alloy in rapid compression tests (ram speed $500 \mathrm{~mm} / \mathrm{s}$, zero soak time) [29].

The peak is believed to originate from the three-dimensional skeletal structure built up in the solid phase at rest, which breaks down under load. The width of the peak (or, more accurately, the downward part of it) is a measure of the time taken to destroy this skeletal structure. A rough 
estimate then gives a breakdown time of about $10 \mathrm{~ms}$, an order of magnitude less than the relaxation times obtained from shear rate jumps in rheometer experiments (see earlier) and must therefore be related to a different mechanism. The height of the peak falls with temperature as the skeletal structure is consumed, and the minimum load beyond the peak also decreases with increasing temperature, both because a more spheroidal microstructure is developed and the fraction liquid increases (see Fig. 5). In practice, successful thixoforming takes place at temperatures where there is little or no peak. Viscosity versus shear rate can be derived from the load-displacement data using a method based on that outlined in Laxmanan and Flemings [12]. As mentioned earlier, this does, however, assume a Newtonian fluid at one stage in the analysis and this may introduce errors.

Data on viscosity versus shear rate for Al-Si alloys is summarised in Fig. 6. It is important to be aware that small changes in silicon content can affect the results quite considerably by changing the solid fraction. The lower values recorded by Yurko and Flemings [28] in comparison with those of

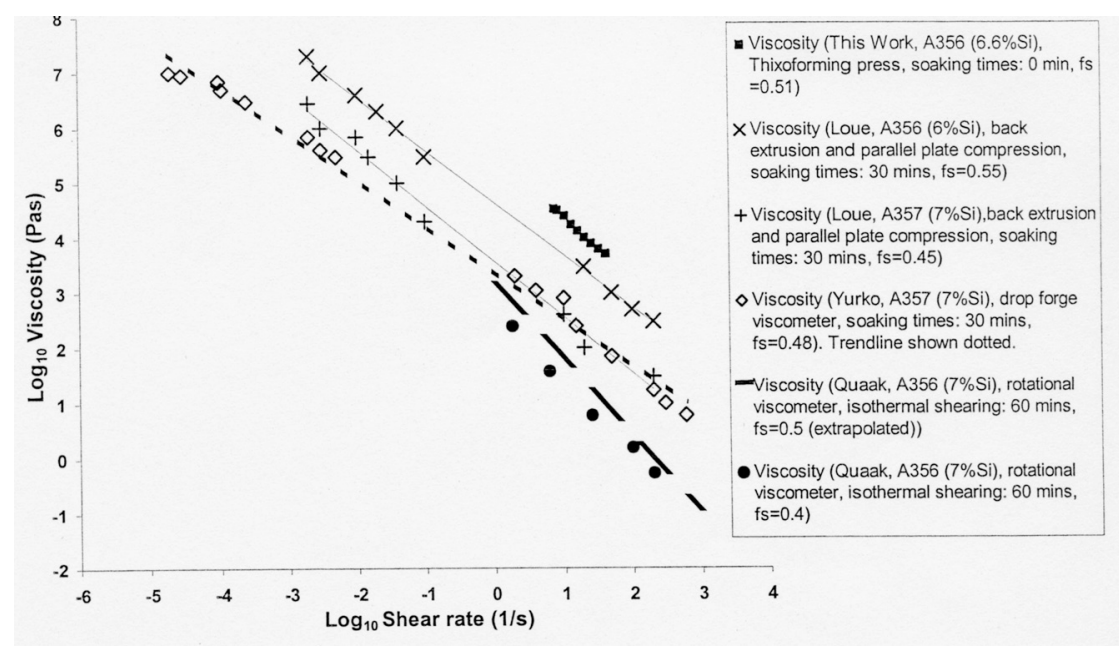

Fig. 6 Comparison of apparent viscosities obtained by various experimental techniques and conditions [29]. ('This work' is [29], 'Loue' is [27], 'Yurko' is [28], 'Quaak' is [15]).
Liu et al. [29] are derived for an alloy with higher silicon content and also one which has been soaked for longer (giving a larger particle size and consequently lower viscosity). Included in the figure is the steady-state viscosity determined by Quaak [15] for a $7 \% \mathrm{Si}$ aluminium alloy, extrapolated to 0.5 fraction solid; this is well below the other results, emphasizing

that the steady state is not achieved in those experiments, nor in industrial thixoforming.

\section{Summary}

In obtaining data for modeling of semi-solid processing, it is important to use both steady-state information and information from transient changes in shear rate. Viscometers offer a measurement volume with a constant shear rate but there are limitations on the shear rate that can be imposed (in comparison with the actual shear rates during semi-solid processing) and until recently high temperature rheometers were rare. In addition, the torque available is generally such that it is difficult to experiment with fractions solid above about 0.5 . Capillary rheometers present difficulties in measuring the pressure drops. Compression between platens is capable of providing data for volume fractions above about 0.5 and for shear rates comparable with those in industrial processes but the shear rate is not uniform and analysis requires simplifying assumptions which may not be justifiable. In summary, the modeler must be careful to understand fully the conditions under which data has been obtained and the potential limitations.

Acknowledgements. The author is grateful to co-workers for discussions, in particular: Dr D H Kirkwood, Dr P J Ward, Dr P Kapranos, Dr S C Hogg and Dr T Y Liu. In addition, she is grateful to the University of Leicester for support in preparing the major review (ref [2]) which has laid the groundwork for this plenary paper. 


\section{References}

1. D.B. Spencer, R. Mehrabian, M.C. Flemings, Metall. Trans. Vol. 3 (1972), p. 1925.

2. H.V. Atkinson, Prog. Mater. Sci. Vol. 50(3) (2005), p.341.

3. D.H. Kirkwood, Proc. $5^{\text {th }}$ Int. Conf. Semi-Solid Processing of Alloys and Composites, Golden Colorado, USA., June 1998, ed. A.K. Bhasin et al., publ. Colorado Scool of Mines, p.xxxiii-xl.

4. H.V. Atkinson, Proc. Int. Conf. Synthesis of Lightweight Metals III, San Diego, 1999, ed. F.H. Froes et al.. publ. Minerals, Metals and Materials Society, p.205-12.

5. A. Alexandrou, Ch. 5 in 'Science and Technology of Semi-Solid Metal Processing', ed. A. de Figueredo, publ. North American Die Casting Association, 2001.

6. Z. Fan, Int. Mater. Rev. Vol. 47 (2002), p.49.

7. M. Modigell, $9^{\text {th }}$ Int. Conf. on Material Forming ESAFORM 2006, Glasgow, 2006, ed. N. Juster and A. Rosochowski, publ. Akapit, Krakow, Poland, 2006, p. 843.

8. A.R.A. McLelland, N.G. Henderson, H.V. Atkinson, D.H. Kirkwood, Mater. Sci. Eng. A, Vol. 232 (1997), p.110.

9. J. Koke, M. Modigell, J. Non-Newtonian Fluid Mech., Vol. 112 (2003), p.141.

10. L. Pape, M. Modigell, $9^{\text {th }}$ Int. Conf. Semi-Solid Processing of Alloys and Composites, Busan, Korea, Sept. 2006.

11. D. Brabazon, D.J. Browne, A.J. Carr, Mater. Sci. Engng., Vol. A326 (2002), p. 370.

12. V. Laxmanan, M.C. Flemings, Met. Trans. A, Vol. 11A (1980), p. 1927.

13. G.H. Nickodemus, C.M. Wang, M.L. Tims, J.J. Fisher, J.J. Cardarella, Proc. $5^{\text {th }}$ Int. Conf. Semi-Solid processing of Alloys and Composites, Golden, Colorado, June 1998, ed. A.K. Bhasin, J.J. Moore, K.P. Young and S. Midson, p. 29.

14. P. Kumar, PhD Thesis, Massachusetts Institute of Technology, Cambridge, Massachusetts, USA, 1994.

15. C.J. Quaak, PhD Thesis, Technische Univesiteit Delft, The Netherlands, 1996.

16. H. Peng, K.K. Wang, Proc. $4^{\text {th }}$ Int. Conf. Semi-Solid Processing of Alloys and Composites, University of Sheffield, Sheffield, 1996, ed. D.H. Kirkwood, P. Kapranos, p.2.

17. M. Mada, F. Ajersch, Mater. Sci. Eng. A, Vol. 212 (1996) p. 157 and p. 171.

18. L. Azzi, F. Ajersch, T.F. Stephenson, Proc. $6^{\text {th }}$ int. Conf. Semi-Solid Processing of Alloys and Composites, Turin, Italy, 2000, Publ. Edimet Spa, ed. G.L. Chiarmetta, M. Rosso, p. 527.

19. M. Modigell, J. Koke, J. Mech Time Dependent Mater., Vol. 3 (1999), p. 15.

20. M. Modigell, J. Koke, J. Mater. Process. Technology, Vol. 111 (2001), p. 53.

21. T.Y. Liu, PhD Thesis, University of Sheffield, Sheffield, UK, 2002.

22. T.Y. Liu, H.V. Atkinson, P.J. Ward, D.H. Kirkwood, Metall. Mater. Trans. A, Vol. 34A (2003), p. 409.

23. L.S. Turng, K.K. Wang, J. Mater. Sci., Vol. 26 (1991), p.2173.

24. M.G. Horsten, C.J. Quaak, W.H. Kool, Proc. $2^{\text {nd }}$ Int. Conf. Semi-Solid Processing of Alloys and Composites, Cambridge, MA, USA, June 1992, publ. Warrendale, TMS, 1992, ed. S.B. Brown, M.C. Flemings, p.359.

25. C.J. Quaak, L. Katgerman, W.H. Kool, p.35 in Ref [16].

26. P. Kumar, C.L. Martin, S.B. Brown, Proc. $3^{\text {rd }}$ Int. Conf. Semi-Solid Processing of Alloys and Composites, Tokyo, Japan, June 1994, Tokyo Institute of Industrial Science, 1994, ed. M. Kiuchi, p. 37.

27. W.R. Loue, M. Suery, J.L. Querbes, p. 266 in Ref [24].

28. J.A. Yurko, M.C. Flemings, Metall. Mater. Trans. A, Vol. 33A (2002), p. 2737.

29. T.Y. Liu, H.V. Atkinson, P. Kapranos, D.H. Kirkwood, S.C. Hogg, Metall. Mater. Trans. A, Vol. 34A (2003), p.1545. 